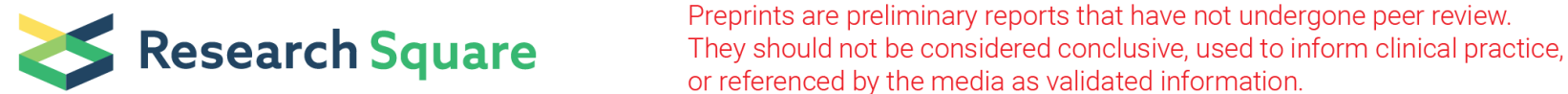 \\ Identification and Characterization of The Gene Profile in Diabetes Induced Erectile Dysfunction Rat Model
}

\section{Zhiguo Zhu}

Affiliated Hospital of Jining Medical University, Jining Medical University

Xiaoli Li

The seventh affiliated hospital (Shenzhen), Sun Yet-sen University

Xiande Cao

Affiliated Hospital of Jining Medical University, Jining Medical University

Huisheng Qin

Affiliated Hospital of Jining Medical University, Jining Medical University

Dong Yue

Affiliated Hospital of Jining Medical University, Jining Medical University

Deqian Liu

Affiliated Hospital of Jining Medical University, Jining Medical University

Guigeng Tan

Affiliated Hospital of Jining Medical University, Jining Medical University

Xujun Xuan

The seventh affiliated hospital (Shenzhen), Sun Yet-sen University

Haizhou Zhu ( $\square$ profzhuhz@163.com )

Affiliated Hospital of Jining Medical University, Jining Medical University

\section{Research Article}

Keywords: Diabetes mellitus, Erectile dysfunction, Gene expression, Molecular signatures, Bioinformatics

Posted Date: March 22nd, 2022

DOI: https://doi.org/10.21203/rs.3.rs-1372202/v2

License: (c) (i) This work is licensed under a Creative Commons Attribution 4.0 International License. Read Full License 


\section{Abstract}

Background: Genes are differentially expressed in diabetes induced erectile dysfunction (DED); however, there is still a lack of systematic and in-depth understanding of the cellular and molecular mechanisms of DED. This study aimed to systematically analyze molecular correlates leading to ED in diabetes by bioinformatics analysis, and provide new insights into the mechanism of DED.

Results: A total of 800 differentially expressed genes (DEGs, 407 upregulated and 393 downregulated) were identified in the diabetic-induced ED samples compared with the control group. The upregulated DEGs were mainly enriched in glucose and lipid metabolism-related pathways, and the downregulated DEGs were primarily enriched in tissue development and structure. The dysregulated extracellular matrix genes (especially related to collagen and elastin) may be closely related to the damage of erectile function of the corpus cavernosum. Sixteen hub genes ( 6 upregulated, 10 downregulated) and 24 modules were detected buy hub gene and MCODE analysis. Protein phosphorylation might play an important role in DED. Furthermore, 20 TFs targeting DEGs were identified by ChEA3.

Conclusions: Our research comprehensively and systematically described the molecular characteristics of DED, and suggested that dysregulated extracellular matrix genes and protein phosphorylation may play critical roles in DED.

\section{Background}

Diabetes mellitus is a chronic, non-communicable disease accompanied by complications of multiple organs and systems [1]. In recent decades, the global incidence of adult diabetes has been increasing, and the growth rate has been accelerating. By 2040 , diabetic patients aged $20-79$ years were expected to increase to 642 million [2]. Diabetes will bring a series of health problems, including sexual dysfunction [3]. In male diabetic patients, sexual dysfunction is mainly manifested as erectile dysfunction (ED). The global prevalence of ED in male diabetes patients overall is $52.5 \%$ (Type I Diabetes: $37.5 \%$, Type II Diabetes: 66.3\%) [4]. In China, the prevalence of ED in men with type 2 diabetes is also as high as $64.2 \%$ [5].

The etiology of diabetic erectile dysfunction (DED) is extremely complicated and may be related to endothelial dysfunction, smooth muscle injury, vascular disease, neuropathy, abnormal hormone levels, inflammation, cavernous fibrosis [6]. For endocrinologists or urologists, DED has always been a huge challenge. Oral phosphodiesterase type-5 inhibitors (PDE5i) are the first-line treatment for ED, including DED. However, DED is often resistant to PDE5i treatment [7]. Intracavernous administration of vasoactive drugs, commonly used as a second-line medical treatment when PDE5i have failed, is often given up due to unavoidable complications, including penile pain, hydrophallus, priapism, fibrosis [7]. Eventually, the patients have to choose penile prosthesis implantation. Therefore, although medicine has made great progress in recent decades, DED is still difficult to treat medically [8]. In addition, research on the mechanism of DED is not uncommon in recent years [9-12], but there is a lack of systematic and profound understanding of the cellular and molecular mechanisms of DED.

Here, we systematically analyzed molecular correlates leading to ED in diabetes by bioinformatics analysis. The differentially expressed genes (DEGs) were identified in diabetes-induced ED rat model. We analyzed the function and interaction pathways of DEGs, and performed hub gene and module analysis. The conserved motifs of the promoter regions and their post-translational phosphorylation modification were studied. In addition, we looked for transcription factors (TFs) that might target DEGs. We present our findings in accordance with the STROBE reporting checklists.

\section{Results}

\section{Identification and enrichment analysis of DEGs}

Array analysis was based on $>1.5$ (or $<0.67$ ) fold change in expression and $P<0.05$. According to these criteria, a total of $800 \mathrm{DEGs}(407$ upregulated and 393 downregulated) were detected (Table S1).

Then, we used Metascape to perform annotation and enrichment analysis of these genes. Figure 1 shows the Metascape enrichment analysis results as a clustered heatmap. In the top 100 enriched clusters (Figure S1), the clusters of upregulated genes and downregulated genes were mostly different, and only a small part of the results overlapped. When the results were limited to top 20 clusters, the overlap probability of enrichment results seemed to increase. However, the main functions of upregulated genes and downregulated of genes were significantly different. The upregulated DEGs were enriched in glucose and lipid metabolism-related pathways: 'fatty acid oxidation', 'monocarboxylic acid metabolic process', 'negative regulation of fatty acid metabolic process' and 'AMPK signaling pathway'. The downregulated DEGs were primarily enriched in tissue development and structure: 'extracellular matrix organization', 'muscle contraction', and 'skeletal system development'. Tables 1 showed the top 20 clusters with their representative enriched terms, the number of genes enriched in each term, and the percentage of genes that are found in the given ontology term.

We further built an enrichment network to demonstrate the relationships between the top 20 terms. In the network, each node represented an enriched cluster. The node was colored first by its cluster ID (Figure 2A) and then by its p-value (Figure 2B). From the network, we found that there were mainly 5 term groups formed: Group 1 - 'extracellular matrix organization' and 'skeletal system development'; Group 2 - 'response to peptide', 'cellular response to hormone stimulus', and 'response to fatty acid'; Group 3 - 'monocarboxylic acid metabolic process', 'purine ribonucleotide metabolic process', 'negative regulation of fatty acid metabolic process', and 'fatty acid oxidation'; Group 4 - 'regulation of protein secretion'; Group 5 - 'regulation of ion transport' and 'muscle contraction'. In Figure 2C, network of enriched terms represented as pie charts, and the color of the pie charts represented the identities of the gene lists (upregulated or downregulated). It could be seen from this network that groups 1 and 5 (related to tissue development and structure) mainly represented downregulated DEGs pathways, and groups 3 and 4 (related to metabolism pathways) were mainly derived from upregulated DEGs. This was consistent with the results reflected in the clustered heatmap (Figure 1). 


\section{PPI network, hub gene and module analysis}

STRING and Cytoscape tool were applied to detect the interactions of the DEGs and construct the PPI network (Figure $3 \mathrm{~A}$ ). Considering the large number of DEGs, we set the minimum required interaction score to 0.7 to avoid the network being too bloated. In the PPI network, the edge color reflected the combined score of the DEGs.

Then, the top 20 hub genes were detected by cytoHubba plug-in used 12 different algorithms (Table 2). Finally, 16 hub genes, detected by more than six algorithms, were selected to build the hub gene PPI network (Figure 3B). Most of the hub genes had a higher degree of connectivity. These genes were enriched in 'post-translational protein phosphorylation' and tissue development - related terms (for example, 'tissue remodeling', 'extracellular matrix organization', 'skeletal system development') (Figure 3C).

MCODE plug-in was used to identify significant modules in the DEGs PPI network, and a total of 24 modules were detected. We selected the top 5 modules to perform analysis using Metascape. Interestingly, we found that all 14 genes in module 1 belong to hub genes. Not surprisingly, module 1 also enriched in 'posttranslational protein phosphorylation' and tissue development - related terms (Figure 4A). It was worth noting that the same 11 genes were enriched in 'posttranslational protein phosphorylation' term: Fst/3, C3, Apob, Men1, Fam20c, Mepe, Fbn1, Igfbp5, Tnc, Msln, Timp1. Thirteen genes belonging to module 2 were enriched in 'protein digestion and absorption' and 'collagen metabolic remodeling' terms (Figure 4B). Module 3 (11 genes) enriched in protein ubiquitination process (Figure 4C). Genes belonging to module 4 regulated signaling pathways involving G proteins (Figure 4D, S2). Module 5 (9 genes) mainly enriched in 'glutathione metabolism' term (Figure 4E, S3).

\section{Identification of conserved motifs in promoter site of genes and prediction of the phosphorylation sites of protein}

Through hub gene and module analysis, we found that the same 11 genes were enriched in 'post-translational protein phosphorylation' pathway. We speculated that this pathway may play an important role in DED, and these genes may have similarities in regulatory patterns. Therefore, we first used GLAM2 tool to identify common conserved motifs in the upstream of these genes. The consensus sequence AAA (G/C) AAA was observed in the promoter site of most studied genes (Figure 5). Then we explored whether the proteins transcribed by these genes have abundant phosphorylation sites. According to the prediction results of NetPhos 3.1 Server, most genes, especially Tnc, Fbn1, C3 and Apob, have abundant phosphorylation sites (Figure 6).

\section{Prediction of TFs associated with DEGs}

TFs are important regulators of gene expression. Through the ChEA3 tool, we found 20 TFs that target upregulated genes or downregulated genes (Figures 7A, 8A). The results showed that TFs related to upregulated genes were mainly concentrated in 'adipose tissue' (Figure 7B), 'thyroid hormone generation', and 'positive regulation of wound healing' (Figure 7C). And TFs related to downregulated genes were mainly concentrated in 'muscle' (Figure 8B) and 'regulation of skeletal muscle tissue development' (Figure 8C).

\section{Discussion}

Penile erection, a complex neurovascular event, is the result of the joint action of nerves, blood vessels, and smooth muscle [13]. The main pathophysiologies of ED are vascular insufficiency and neuropathy. Since diabetes, a metabolic disorder characterized by hyperglycemia, can cause neurological and vascular disease, men with diabetes are at risk of ED. In fact, $52.5 \%$ of patients suffer from ED after the diagnosis of diabetes. Type 2 diabetes (T2DM) accounts for $90 \% \sim 95 \%$ [14] of diabetes cases, and the incidence of ED in male patients with type 2 diabetes exceeds $60 \%$. Unfortunately, the existing treatments for ED are not very effective for DED. Considering the huge number of diabetic patients, DED places a huge burden on individuals, families, and society. The difficulty of DED treatment is that the pathogenesis is complicated and unclear. Hence, identifying underlying cellular and molecular mechanisms in DED is important for improving diagnostic and therapeutic strategy.

Although previous studies [15-17] have reported changes in the gene expression profile of DED cavernous tissues, the analysis methods of these studies are relatively simple. In this study, we systematically analyzed molecular correlates leading to DED by bioinformatics analysis. Through the GEO2R tool, 800 DGEs were identified. Changes in the expression of a large number of genes also indicate the complexity of the cause of DED. To further explore the relationships between them, we performed enrichment analysis, built a PPI network, did hub gene and module analysis, identified conserved motifs in promoter site, and predicted the phosphorylation sites. Finally, we predicted TFs associated with DEGs.

The results demonstrated that upregulated genes were mainly involved in lipid metabolism-related pathways. This was consistent with the fact that DED patients suffer from diabetes. For example, Mitochondrial 3-hydroxy-3-methylglutaryl-CoA synthase (Hmgcs2), which catalyzes the first reaction of ketogenesis [18], was the one with the highest fold change (31.40) in upregulated DEGs. Studies have shown that Hmgcs2 was closely related to diabetes [19, 20], and inhibiting Hmgcs2 could alleviate DED [17]. Ceruloplasmin ( $C p)$ increased in diabetes [15, 21], and inhibited the activation of endothelial nitric oxide synthase [22]. The downregulated DEGs were primarily enriched in tissue development and structure: 'extracellular matrix organization', 'muscle contraction', and 'skeletal system development'. 'Extracellular matrix organization' was the most statistically significant term for enrichment analysis, and 24 downregulated genes were enriched in this term. Collagen, elastin, and proteoglycan constitute the extracellular matrix of human and rat erectile tissues. The important pathological changes of ED are the apoptosis of cavernous smooth muscle cells, the change of the ratio of smooth muscle to collagen, and the atrophy of endothelial cells. Therefore, changes in the composition and structure of the extracellular matrix caused by diabetes may impair erectile function. We found that the downregulated genes included collagens type $1,2,3,5,6,11,14,15$. Collagen types $1,2,3,5$, and 11 were the fibril forming collagen [23]. Among them, collagen type 1 is the most abundant form of collagen in erectile tissues of humans and rats, and constitutes the framework of the cavernous matrix structure. Collagen 1 is usually formed by two identical a1(I)-chains (Col1a1) and one a2(I)-chain (Col1a2), and provides tensile stiffness in most organs. In our analysis, both Col1a1 and Col1a2 have decreased expression in DED, which would inevitably affect the erectile function of the penis.

Page 3/11 
Furthermore, genes associated with elastic fibers (Lox [24], Fbn1 [25], and so on) were also decreased. Different from other studies, we also built an enrichment network to demonstrate the relationships between the enriched terms. Terms with similar functions were also gathered: groups 1 and 5 - related to tissue development and structure, groups 3 and 4 - related to metabolism pathways. These all shown close molecular connections in DEGs.

In the PPI network, we found that there were rich interactions between the most DEGs. Sixteen hub genes ( 6 upregulated, 10 downregulated) were selected by 12 algorithms in cytoHubba plug-in. Interesting, these genes were enriched in 'post-translational protein phosphorylation' and terms regarding tissue development, not lipid metabolism-related pathways.

MCODE can find and extract the closely related regions in the PPI network, and a total of 24 modules were detected. We selected the top 5 modules for further analysis. It is worth noting that the genes in module 1 (14 genes) all belong to hub genes. The results of module 1 enrichment analysis were also similar to that of hub genes. The role of these genes in DED deserves further study. In addition, we found that 'post-translational protein phosphorylation' was the most statistically significant term for enrichment analysis in module 1 and hub genes, and the same 11 genes were enriched in this term. To verify whether these genes have similar regulatory patterns, we used GLAM2 tool to identify common conserved motifs in the upstream of them. In this study, AAA - rich conserved motifs was detected in the promoter site of these genes affecting the expression patterns by activating and suppressing the transcription of studied genes. We also found that the proteins transcribed by these genes have abundant phosphorylation sites. Protein phosphorylation is one of the most common and important post-translational modifications, and it is an important cellular regulatory mechanism [26]. Abnormal protein phosphorylation can be found in many diseases, and the strategy of activating or inhibiting this process to treat diseases is theoretically feasible. There are relatively few studies on protein phosphorylation in the occurrence and progression of ED. Module 2 mainly enriched signatures regarding collagen metabolism. From the perspective of enrichment analysis, module 1 and module 2 represented downregulated genes. It is very interesting that both the enrichment analysis of hub genes and modules lacks terms regarding glucose and lipid metabolism. Perhaps genes related to glucose and lipid metabolism do not play a key role in penile lesions, and just reflected the body's metabolic disorders. Because despite good control of blood glucose level, ED is still getting worse in some DED patients [27].

TFs are a group of proteins that ensure that the target genes are expressed at a specific time and space with a specific intensity. They are important regulators of gene expression. We predicted TFs associated with DEGs used the ChEA3 tool. By analyzing the tissue specificity and function of these TFs, we found that TFs targeting upregulated genes were closely related to glucose and lipid metabolism, and TFs targeting downregulated genes were more related to tissue development. These were consistent with the enrichment results of DEGs. Studies have reported that some of these TFs, (for example, Foxa1 [28], ELF3 [29], Tbx15 [30], TWIST1, TWIST2 [31]) were involved in diabetes or muscle development. But the role of these TFs in DED has not been studied.

Although we got meaningful results, there are several noteworthy limitations in our study. First, sequencing data comes from rat samples. Although some genes are conserved between species, species differences are a key issue that cannot be ignored. In the future, the findings of this study should be verified in human tissues. Cavernous tissue from men undergoing penile surgery or brain-dead male organ donors is an ideal solution [32]. Second, the valuable genes discovered in this study need to be experimentally verified to explore their mechanism in DED. Third, this study only involves changes in mRNA expression, lack of the data of non-coding RNA (miRNA, IncRNA, and circRNA). Non-coding RNA plays an important role in the occurrence and development of diabetes and ED. [9, 33-35] We can reveal molecular mechanisms of DED more deeply by constructing potential IncRNA-miRNA-mRNA regulatory network.

\section{Conclusions}

In this study, we successfully defined molecular signatures in DED. We found that 800 DEGs (407 upregulated and 393 downregulated) in the diabetic-induced ED samples compared with the control group. The upregulated DEGs were enriched in glucose and lipid metabolism-related pathways, and the downregulated DEGs were primarily enriched in tissue development and structure. The dysregulated extracellular matrix genes (especially related to collagen and elastin) may be closely related to the damage of erectile function of the corpus cavernosum. Through hub gene and module analysis, we found some genes that may play a key role in the development of DED, the role of protein phosphorylation in DED needs to be taken seriously.

\section{Methods}

\section{Data collection}

We used "diabetes" and "erectile dysfunction" to search the gene expression datasets in the Gene Expression Omnibus (GEO) database (https://www.ncbi.nlm.nih.gov/geo/). Considering ethical principles, it is extremely difficult to obtain penile tissue samples from DED patients. Finally, GSE2457 was selected for the next analysis [15]. In this study, researchers performed microarray experiments on the cavernous tissue of the diabetes-induced ED rat model.

\section{Identification of differentially expressed genes}

The GEO2R tool (https://www.ncbi.nlm.nih.gov/geo/geo2r/) was used to extract differentially expressed genes (DEGs). $P<0.05$ and fold change > 1.5 (or < 0.67) were chosen as the cutoff criteria.

\section{Gene annotation and enrichment analysis of DEGs}

Metascape [36] (http://metascape.org), a web-based tool, can provide automated gene list annotation and enrichment analysis. Enrichment analysis was based on the following ontology sources: gene ontology (GO) processes, KEGG pathways, canonical pathways, Reactome gene sets, PaGenBase and CORUM. In addition, we used this tool to draw the heatmap and network of the enriched terms.

PPI network, hub gene and module analysis

Page $4 / 11$ 
Search Tool for the Retrieval of Interacting Genes [37] (STRING, https://string-db.org/) was applied to detect the interactions of the DEGs and construct the PPI network. Cytoscape software [38] was used to optimize the PPI network. Two Cytoscape software plug-ins, cytoHubba and Molecular Complex Detection (MCODE), were used to identify hub genes and screen modules of the PPI network.

\section{Identification of conserved motifs in promoter site}

Ensembl [39] (https://ensembl.org/) was used to obtain the promoter site (2000 bp upstream of transcription start site). Gapped Local Alignment of Motifs [40] (GLAM2) was used to discover common conserved motifs in the gene of interest.

\section{Prediction of the phosphorylation sites}

The protein sequence was retrieved from the Protein (https://www.ncbi.nlm.nih.gov/protein). NetPhos 3.1 Server [41]

(http://www.cbs.dtu.dk/services/NetPhos/) was used to predict serine, threonine and tyrosine phosphorylation sites in selected proteins.

\section{Prediction of TFs associated with DEGs}

ChEA3 (https://maayanlab.cloud/chea3/) is an online tool that can be used for transcription factor enrichment analysis by orthogonal omics integration [42]. We used this tool to find TFs targeting DEGs and annotate these TFs.

\section{Statistical analysis}

All the statistical analyses were conduct with SPSS version 20.0 (SPSS Inc., Chicago, IL, USA) and GraphPad Prism version 8.0 (GraphPad Software, San Diego, CA, USA). $P<0.05$ indicated statistical significance.

\section{Abbreviations}

DED diabetes induced erectile dysfunction

DEGs differentially expressed genes

PPI protein-protein interaction

TFs transcription factors

ED erectile dysfunction

PDE5i phosphodiesterase type-5 inhibitors

GEO Gene Expression Omnibus

GO gene ontology

T2DM Type 2 diabetes

\section{Declarations}

Ethics approval and consent to participate: Not applicable.

Consent for publication: Not applicable.

Availability of data and material: The data can be found in the Gene Expression Omnibus (GEO) database (https://www.ncbi.nlm.nih.gov/geo/, GSE2457).

Competing interests: The authors declare that they have no competing interests.

Funding: This work was supported by PhD Research Foundation of Affiliated Hospital of Jining Medical University (2022-BS-001) and Guangdong Basic and Applied Basic Research Foundation (2020A1515110946).

Author's contributions: (I) Conception and design: Z Zhu, X Xuan; (II) Administrative support: H Zhu, X Xuan, and Z Zhu; (III) Provision of study materials or patients: H Zhu, Z Zhu; (IV) Collection and assembly of data: Z Zhu, X Li, X Cao, D Liu; (V) Data analysis and interpretation: Z Zhu, X Li, Y Dong, G Tan; (VI) Manuscript writing: All authors; (VII) Final approval of manuscript: All authors.

Acknowledgements: Not applicable.

\section{References}

1. Hua F: New insights into diabetes mellitus and its complications: a narrative review. Annals of translational medicine 2020, 8(24):1689.

2. Ogurtsova K, da Rocha Fernandes JD, Huang Y, Linnenkamp U, Guariguata L, Cho NH, Cavan D, Shaw JE, Makaroff LE: IDF Diabetes Atlas: Global estimates for the prevalence of diabetes for 2015 and 2040. Diabetes research and clinical practice 2017, 128:40-50. 
3. Zhang X, Yang B, Li N, Li H: Prevalence and Risk Factors for Erectile Dysfunction in Chinese Adult Males. The journal of sexual medicine 2017, 14(10):1201-1208.

4. Kouidrat Y, Pizzol D, Cosco T, Thompson T, Carnaghi M, Bertoldo A, Solmi M, Stubbs B, Veronese N: High prevalence of erectile dysfunction in diabetes: a systematic review and meta-analysis of 145 studies. Diabetic medicine : a journal of the British Diabetic Association 2017, 34(9):1185-1192.

5. Xu Y, Zhang Y, Yang Y, Liu L, Chen Y, Liu X: Prevalence and correlates of erectile dysfunction in type 2 diabetic men: a population-based cross-sectional study in Chinese men. International journal of impotence research 2019, 31(1):9-14.

6. Cellek S, Cameron NE, Cotter MA, Muneer A: Pathophysiology of diabetic erectile dysfunction: potential contribution of vasa nervorum and advanced glycation endproducts. International journal of impotence research 2013, 25(1):1-6.

7. Hatzimouratidis $\mathrm{K}$, Hatzichristou D: How to treat erectile dysfunction in men with diabetes: from pathophysiology to treatment. Current diabetes reports 2014, 14(11):545.

8. Malavige LS, Levy JC: Erectile dysfunction in diabetes mellitus. The journal of sexual medicine 2009, 6(5):1232-1247.

9. Huo W, Li Y, Zhang Y, Li H: Mesenchymal stem cells-derived exosomal microRNA-21-5p downregulates PDCD4 and ameliorates erectile dysfunction in a rat model of diabetes mellitus. FASEB journal : official publication of the Federation of American Societies for Experimental Biology 2020, 34(10):1334513360.

10. Che D, Fang Z, Yan L, Du J, Li F, Xie J, Feng J, Yin P, Qi W, Yang Z et al: Elevated pigment epithelium-derived factor induces diabetic erectile dysfunction via interruption of the Akt/Hsp90ß/eNOS complex. Diabetologia 2020, 63(9):1857-1871.

11. Yuan P, Ma D, Gao X, Wang J, Li R, Liu Z, Wang T, Wang S, Liu J, Liu X: Liraglutide Ameliorates Erectile Dysfunction via Regulating Oxidative Stress, the RhoA/ROCK Pathway and Autophagy in Diabetes Mellitus. Frontiers in pharmacology 2020, 11:1257.

12. Wen Y, Liu G, Zhang Y, Li H: MicroRNA-205 is associated with diabetes mellitus-induced erectile dysfunction via down-regulating the androgen receptor. Journal of cellular and molecular medicine 2019, 23(5):3257-3270.

13. Gratzke C, Angulo J, Chitaley K, Dai YT, Kim NN, Paick JS, Simonsen U, Uckert S, Wespes E, Andersson KE et al: Anatomy, physiology, and pathophysiology of erectile dysfunction. The journal of sexual medicine 2010, 7(1 Pt 2):445-475.

14. Chen L, Magliano DJ, Zimmet PZ: The worldwide epidemiology of type 2 diabetes mellitus-present and future perspectives. Nature reviews Endocrinology 2011, 8(4):228-236

15. Sullivan CJ, Teal TH, Luttrell IP, Tran KB, Peters MA, Wessells H: Microarray analysis reveals novel gene expression changes associated with erectile dysfunction in diabetic rats. Physiological genomics 2005, 23(2):192-205.

16. Kam SC, Lee SH, Jeon JH, So I, Chae MR, Park JK, Lee SW: Gene expression profile comparison in the penile tissue of diabetes and cavernous nerve injury-induced erectile dysfunction rat model. Investigative and clinical urology 2016, 57(4):286-297.

17. Zhang Z, Zhang HY, Zhang Y, Li H: Inactivation of the Ras/MAPK/PPARY signaling axis alleviates diabetic mellitus-induced erectile dysfunction through suppression of corpus cavernosal endothelial cell apoptosis by inhibiting HMGCS2 expression. Endocrine 2019, 63(3):615-631.

18. Newman JC, Verdin E: Ketone bodies as signaling metabolites. Trends in endocrinology and metabolism: TEM 2014, 25(1):42-52.

19. Kurtz CL, Peck BC, Fannin EE, Beysen C, Miao J, Landstreet SR, Ding S, Turaga V, Lund PK, Turner S et al: MicroRNA-29 fine-tunes the expression of key FOXA2-activated lipid metabolism genes and is dysregulated in animal models of insulin resistance and diabetes. Diabetes 2014, 63(9):3141-3148.

20. Cook GA, Lavrentyev EN, Pham K, Park EA: Streptozotocin diabetes increases mRNA expression of ketogenic enzymes in the rat heart. Biochimica et biophysica acta General subjects 2017, 1861(2):307-312.

21. Gerhardinger C, Costa MB, Coulombe MC, Toth I, Hoehn T, Grosu P: Expression of acute-phase response proteins in retinal Müller cells in diabetes. Investigative ophthalmology \& visual science 2005, 46(1):349-357.

22. Bianchini A, Musci G, Calabrese L: Inhibition of endothelial nitric-oxide synthase by ceruloplasmin. The Journal of biological chemistry 1999 , 274(29):20265-20270.

23. Gelse K, Pöschl E, Aigner T: Collagens-structure, function, and biosynthesis. Advanced drug delivery reviews 2003, 55(12):1531-1546.

24. Song YL, Ford JW, Gordon D, Shanley CJ: Regulation of lysyl oxidase by interferon-gamma in rat aortic smooth muscle cells. Arteriosclerosis, thrombosis, and vascular biology 2000, 20(4):982-988.

25. Sakai LY, Keene DR, Renard M, De Backer J: FBN1: The disease-causing gene for Marfan syndrome and other genetic disorders. Gene 2016, 591(1):279291.

26. Ardito F, Giuliani M, Perrone D, Troiano G, Lo Muzio L: The crucial role of protein phosphorylation in cell signaling and its use as targeted therapy (Review). International journal of molecular medicine 2017, 40(2):271-280.

27. Wang L, Xu Y, Li H, Lei H, Guan R, Gao Z, Xin Z: Antioxidant icariside II combined with insulin restores erectile function in streptozotocin-induced type 1 diabetic rats. Journal of cellular and molecular medicine 2015, 19(5):960-969.

28. Vatamaniuk MZ, Gupta RK, Lantz KA, Doliba NM, Matschinsky FM, Kaestner KH: Foxa1-deficient mice exhibit impaired insulin secretion due to uncoupled oxidative phosphorylation. Diabetes 2006, 55(10):2730-2736.

29. Lopes M, Kutlu B, Miani M, Bang-Berthelsen CH, Størling J, Pociot F, Goodman N, Hood L, Welsh N, Bontempi G et al: Temporal profiling of cytokineinduced genes in pancreatic $\beta$-cells by meta-analysis and network inference. Genomics 2014, 103(4):264-275.

30. Lee KY, Singh MK, Ussar S, Wetzel P, Hirshman MF, Goodyear LJ, Kispert A, Kahn CR: Tbx15 controls skeletal muscle fibre-type determination and muscle metabolism. Nature communications 2015, 6:8054. 
31. Mudry JM, Massart J, Szekeres FL, Krook A: TWIST1 and TWIST2 regulate glycogen storage and inflammatory genes in skeletal muscle. The Journal of endocrinology 2015, 224(3):303-313.

32. Wessells H, Sullivan CJ, Tsubota Y, Engel KL, Kim B, Olson NE, Thorner D, Chitaley K: Transcriptional profiling of human cavernosal endothelial cells reveals distinctive cell adhesion phenotype and role for claudin 11 in vascular barrier function. Physiological genomics 2009, 39(2):100-108.

33. Cong R, Wang Y, Wang Y, Zhang Q, Zhou X, Ji C, Yao L, Song N, Meng X: Comprehensive Analysis of IncRNA Expression Pattern and IncRNA-miRNA-mRNA Network in a Rat Model With Cavernous Nerve Injury Erectile Dysfunction. The journal of sexual medicine 2020, 17(9):1603-1617.

34. Ying W, Gao H, Dos Reis FCG, Bandyopadhyay G, Ofrecio JM, Luo Z, Ji Y, Jin Z, Ly C, Olefsky JM: MiR-690, an exosomal-derived miRNA from M2-polarized macrophages, improves insulin sensitivity in obese mice. Cell metabolism 2021.

35. Peng F, Gong W, Li S, Yin B, Zhao C, Liu W, Chen X, Luo C, Huang Q, Chen T et al: circRNA_010383 Acts as a Sponge for miR-135a, and Its Downregulated Expression Contributes to Renal Fibrosis in Diabetic Nephropathy. Diabetes 2021, 70(2):603-615.

36. Zhou Y, Zhou B, Pache L, Chang M, Khodabakhshi AH, Tanaseichuk O, Benner C, Chanda SK: Metascape provides a biologist-oriented resource for the analysis of systems-level datasets. Nature communications 2019, 10(1):1523.

37. Szklarczyk D, Gable AL, Lyon D, Junge A, Wyder S, Huerta-Cepas J, Simonovic M, Doncheva NT, Morris JH, Bork P et al: STRING v11: protein-protein association networks with increased coverage, supporting functional discovery in genome-wide experimental datasets. Nucleic acids research 2019 , 47(D1):D607-d613.

38. Shannon P, Markiel A, Ozier O, Baliga NS, Wang JT, Ramage D, Amin N, Schwikowski B, Ideker T: Cytoscape: a software environment for integrated models of biomolecular interaction networks. Genome research 2003, 13(11):2498-2504.

39. Yates AD, Achuthan P, Akanni W, Allen J, Allen J, Alvarez-Jarreta J, Amode MR, Armean IM, Azov AG, Bennett R et al: Ensembl 2020. Nucleic acids research 2020, 48(D1):D682-d688.

40. Frith MC, Saunders NF, Kobe B, Bailey TL: Discovering sequence motifs with arbitrary insertions and deletions. PLoS computational biology 2008, 4(4):e1000071.

41. Blom N, Sicheritz-Pontén T, Gupta R, Gammeltoft S, Brunak S: Prediction of post-translational glycosylation and phosphorylation of proteins from the amino acid sequence. Proteomics 2004, 4(6):1633-1649.

42. Keenan AB, Torre D, Lachmann A, Leong AK, Wojciechowicz ML, Utti V, Jagodnik KM, Kropiwnicki E, Wang Z, Ma'ayan A: ChEA3: transcription factor enrichment analysis by orthogonal omics integration. Nucleic acids research 2019, 47(W1):W212-w224.

\section{Tables}

Table 1 Top 20 clusters with their representative enriched terms

\begin{tabular}{|c|c|c|c|c|c|c|c|}
\hline Gene list & Terms & Category & Description & Count & $\%$ & $\log 10(\mathrm{P})$ & $\log 10(q)$ \\
\hline $\mathbf{\square}$ & R-RNO-1474244 & Reactome Gene Sets & Extracellular matrix organization & 24 & 6.78 & -11.15 & -6.90 \\
\hline $\mathbf{\square}$ & GO:0032787 & GO Biological Processes & monocarboxylic acid metabolic process & 61 & 8.43 & -10.44 & -6.16 \\
\hline $\mathbf{\square}$ & R-RNO-397014 & Reactome Gene Sets & Muscle contraction & 20 & 5.65 & -10.23 & -6.90 \\
\hline $\mathbf{\square}$ & GO:1901652 & GO Biological Processes & response to peptide & 63 & 8.70 & -9.72 & -5.74 \\
\hline $\mathbf{\square}$ & GO:0043269 & GO Biological Processes & regulation of ion transport & 68 & 9.39 & -9.36 & -5.74 \\
\hline $\mathbf{\square}$ & GO:0019395 & GO Biological Processes & fatty acid oxidation & 16 & 4.31 & -9.27 & -6.06 \\
\hline $\mathbf{\square}$ & GO:0048871 & GO Biological Processes & multicellular organismal homeostasis & 53 & 7.32 & -9.25 & -5.74 \\
\hline $\mathbf{\square}$ & GO:0009611 & GO Biological Processes & response to wounding & 61 & 8.43 & -9.05 & -5.73 \\
\hline $\mathbf{\square}$ & GO:0050708 & GO Biological Processes & regulation of protein secretion & 28 & 7.55 & -7.56 & -4.84 \\
\hline $\mathbf{\square}$ & GO:0032870 & GO Biological Processes & cellular response to hormone stimulus & 64 & 8.84 & -7.51 & -4.65 \\
\hline $\mathbf{\square}$ & GO:0009150 & GO Biological Processes & purine ribonucleotide metabolic process & 45 & 6.22 & -7.47 & -4.63 \\
\hline $\mathbf{\square}$ & GO:0002931 & GO Biological Processes & response to ischemia & 15 & 2.07 & -7.31 & -4.52 \\
\hline $\mathbf{\square}$ & ko04152 & KEGG Pathway & AMPK signaling pathway & 20 & 2.76 & -7.02 & -4.33 \\
\hline $\mathbf{\square}$ & GO:0001501 & GO Biological Processes & skeletal system development & 30 & 8.47 & -6.70 & -4.12 \\
\hline $\mathbf{\square}$ & GO:0045922 & GO Biological Processes & negative regulation of fatty acid metabolic process & 8 & 2.16 & -6.63 & -4.10 \\
\hline $\mathbf{\square}$ & GO:0010038 & GO Biological Processes & response to metal ion & 45 & 6.22 & -6.34 & -3.84 \\
\hline $\mathbf{\square}$ & GO:0070848 & GO Biological Processes & response to growth factor & 60 & 8.29 & -5.97 & -3.54 \\
\hline $\mathbf{\square}$ & GO:0032526 & GO Biological Processes & response to retinoic acid & 14 & 3.95 & -5.92 & -3.54 \\
\hline $\mathbf{\square}$ & GO:0070542 & GO Biological Processes & response to fatty acid & 19 & 2.62 & -5.87 & -3.47 \\
\hline $\mathbf{\square}$ & GO:0001659 & GO Biological Processes & temperature homeostasis & 23 & 3.18 & -5.78 & -3.39 \\
\hline
\end{tabular}

Abbreviations: $\square$, upregulated genes; $\square$, downregulated genes; GO, gene ontology; KEGG, Kyoto Encyclopedia of Genes and Genomes; AMPK, Adenosine 5'-monophosphate -activated protein kinase

Table 2 Top 20 hub genes detected using the 12 algorithms in cytoHubba plug-in 


\begin{tabular}{|c|c|c|c|c|c|c|c|c|c|c|c|c|}
\hline & MCC & DNMC & MNC & Degree & EPC & BottleNeck & EcCentricity & Closeness & Radiality & Betweenness & Stress & 1 \\
\hline 1 & $\mathrm{C} 3$ & Fam20c & Apob & Fn1 & Col1a1 & Fn1 & Fbxo32 & Fn1 & Fn1 & Fn1 & Fn1 & 1 \\
\hline 2 & Fn1 & Mepe & Fn1 & Apob & Fn1 & Apob & Anapc7 & Apob & Apob & Hras & Apob & $؛$ \\
\hline 3 & Fbn1 & Fstl3 & Fbn1 & $\mathrm{C} 3$ & Msln & Hras & Kctd6 & Fbn1 & Fstl3 & Fbxo32 & Fbxo32 & I \\
\hline 4 & Timp1 & $\operatorname{lgfbp5}$ & Col1a1 & Fbn1 & Fbn1 & Fbxo32 & Fbxo2 & Cp & Cp & Apob & Foxo3 & $\vdots$ \\
\hline 5 & Msln & Men1 & Col3a1 & Col1a1 & Col5a1 & Mstn & Ubac1 & $\mathrm{C} 3$ & Fbn1 & Foxo3 & Hras & I \\
\hline 6 & Apob & Tnc & Col1a2 & Timp1 & Apob & Fstl3 & Asb2 & Timp1 & Timp1 & C3 & Fasn & I \\
\hline 7 & Cp & $\mathrm{C} 3$ & Timp1 & Col3a1 & Cp & Calm1 & Rnf213 & Msln & Msln & Cat & Cops5 & I \\
\hline 8 & Men1 & Col15a1 & Msln & Col1a2 & C3 & Cp & Rbbp6 & Fstl3 & $\mathrm{C} 3$ & Mstn & Me1 & 1 \\
\hline 9 & Vcan & Col14a1 & Col5a2 & $\mathrm{Cp}$ & Col3a1 & Ins2 & Lnx1 & Men1 & Men1 & Calm1 & Cat & 1 \\
\hline 10 & Fstl3 & Anapc7 & Col5a1 & Msln & Timp1 & Srebf1 & $\mathrm{Fbxo21}$ & Hras & Ctnnb1 & Fstl3 & Ldhc & - \\
\hline 11 & Fam20c & Ubac1 & Col2a1 & Hras & Tnc & Ceacam1 & Rnf7 & Vcan & Vcan & Psmb8 & Mstn & 1 \\
\hline 12 & Mepe & Rnf213 & Cp & Fbxo32 & Col1a2 & $\mathrm{C} 3$ & Ankrd9 & Fam20c & Fam20c & Cops5 & Cp & I \\
\hline 13 & $\operatorname{lgfbp} 5$ & Rbbp6 & Vcan & Men1 & Mepe & Foxo3 & Cops5 & Mepe & Mepe & Ctnnb1 & Fstl3 & 1 \\
\hline 14 & Tnc & $\operatorname{Lnx1}$ & Fam20c & Col5a2 & Igfbp5 & Fbn1 & Myh1 & $\operatorname{lgfbp} 5$ & $\operatorname{lgfbp} 5$ & Ceacam1 & $\mathrm{C} 3$ & I \\
\hline 15 & Col1a1 & Col4a3 & Mepe & Col5a1 & Col5a2 & Cat & Foxo3 & Tnc & Tnc & $\mathrm{Cp}$ & Xab2 & 1 \\
\hline 16 & Col3a1 & Col11a1 & Fstl3 & Col2a1 & Col2a1 & Pak3 & Ring1 & Ceacam1 & Hras & Fasn & Psmb8 & 1 \\
\hline 17 & Col1a2 & Col5a3 & Sparc & Ceacam1 & Sparc & Cops5 & Mstn & Col1a1 & Cat & Anapc7 & Aldoc & I \\
\hline 18 & Col5a2 & $\mathrm{Cp}$ & Igfbp5 & Fstl3 & Men1 & Arfgap1 & Prkaa2 & Sparc & Ceacam1 & Me1 & Fbn1 & 1 \\
\hline 19 & Col5a1 & Vcan & Men1 & Vcan & Fam20c & Mapk6 & Tnnt2 & Col1a2 & Foxo3 & Espl1 & Hnrnpa2b1 & 1 \\
\hline 20 & Col2a1 & Serpinh1 & Tnc & Calm1 & Fstl3 & Fasn & Casq1 & Col3a1 & Mstn & Ldhc & Calm1 & I \\
\hline
\end{tabular}

\section{Figures}

\section{Figure 1}

Heatmap of enriched terms across input gene lists.

\section{Figure 2}

Visualizations of functional enrichment and interactome analysis results of DEGs. Network of enriched terms: (A) colored by enriched terms and 5 term groups formed, (B) colored by $P$ value, (C) color-coded based on the identities of the gene lists.

Abbreviations: DEGs, differentially expressed genes; GO, gene ontology; AMPK, Adenosine 5'-monophosphate -activated protein kinase. 


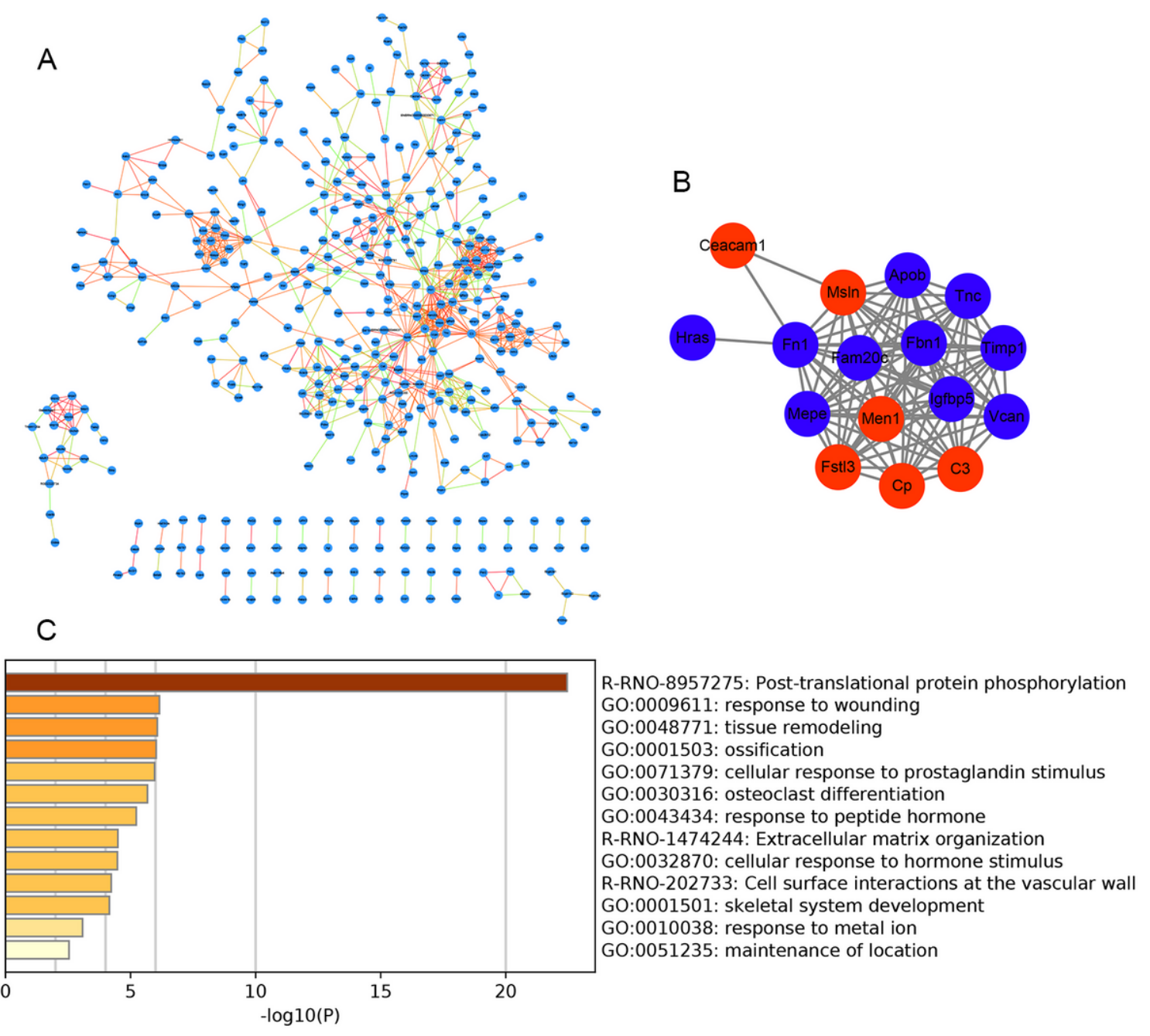

Figure 3

(A) PPI network of DEGs. The edge color reflected the combined score of the genes (green $\rightarrow$ red $=$ low score $\rightarrow$ high score). (B) PPI network of 16 hub genes, and enrichment analysis of these genes. Abbreviations: DEGs, differentially expressed genes; GO, gene ontology; PPI, protein - protein interaction. 


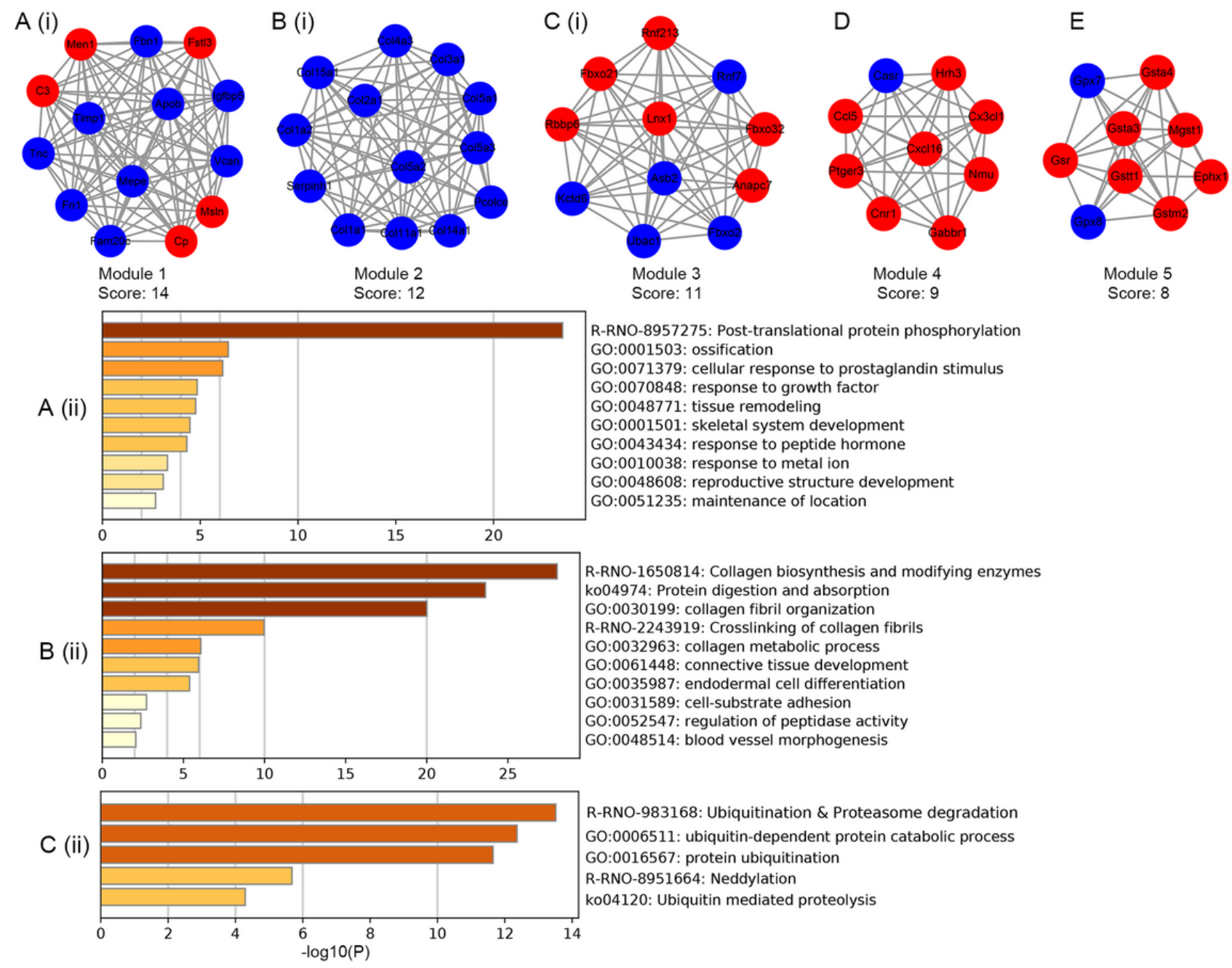

Figure 4

Module analysis of DEGs. Top 5 modules screened from the PPI network. The genes of the top 3 modules were performed with enrichment analysis. Nodes were color-coded based on the expression of DEGs (red = upregulated; blue = downregulated). Abbreviations: DEGs, differentially expressed genes; PPI, protein - protein interaction; GO, gene ontology.

NAME START

Fstl3

C3

Apob

$\mathrm{N} \operatorname{Ton} 1$
1116

553

1072
11

\section{SITES}

a a a a a g a c c a a a a a a a a a a a a a a a . a

a a a a a a a a a a a a a a a a a a a a a a c

a c a c a c a c a c a c a c a c a c a c a c a c a c a c a c a c a c

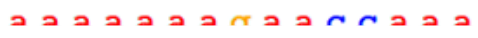

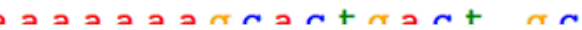

END

1084

585

1105 


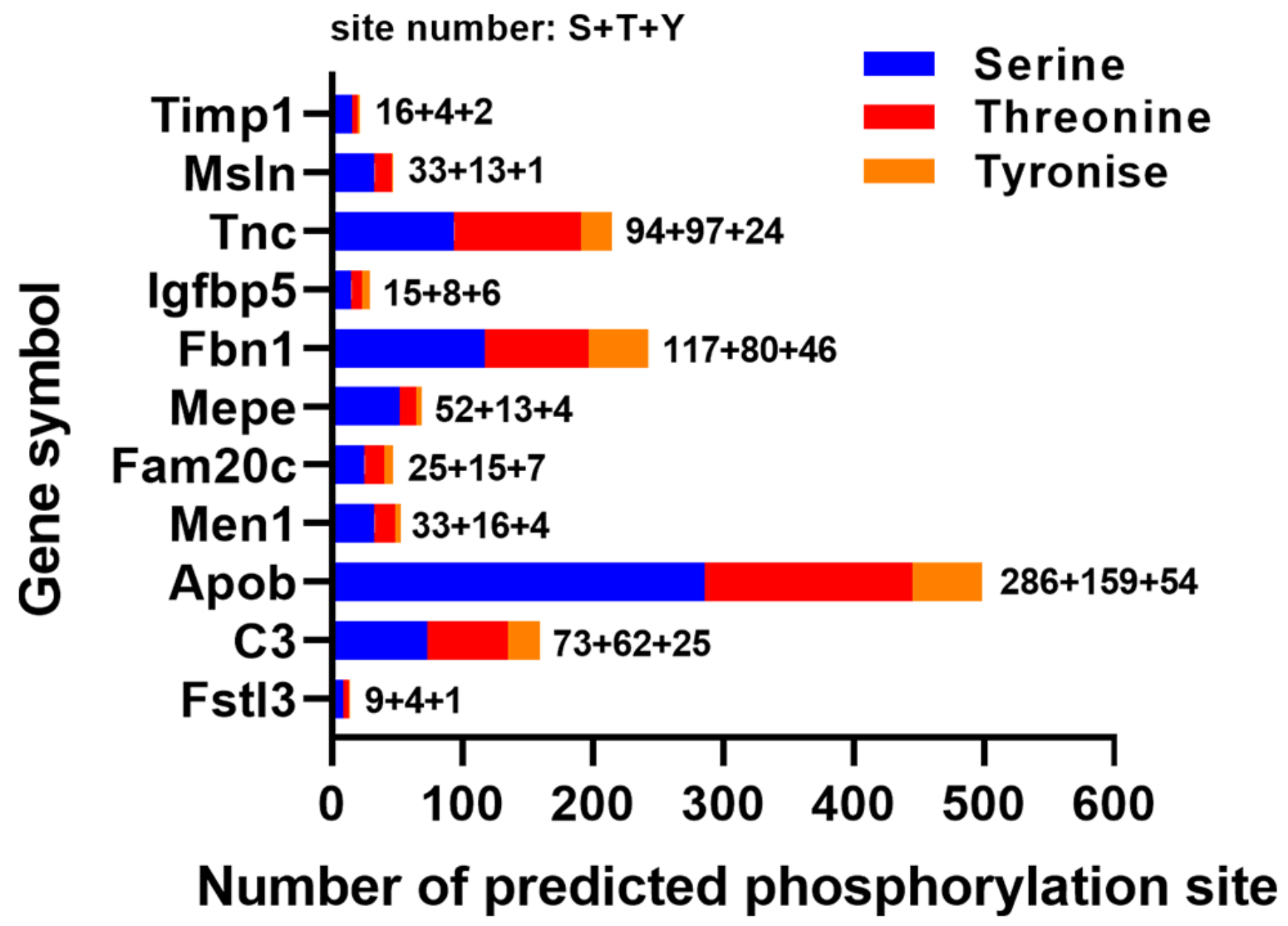

Figure 6

The prediction of phosphorylation sites of proteins transcribed by selected genes.

Figure 7

The prediction of TFs related to upregulated genes. (A) top 10 TFs targeting upregulated genes; (B) the tissue locations of predicted TFs; (C) the biological functions of predicted TFs. Abbreviations: TFs, transcriptional factors.

Figure 8

The prediction of TFs related to downregulated genes. (A) top 10 TFs targeting downregulated genes; (B) the tissue locations of predicted TFs; (C) the biological functions of predicted TFs. Abbreviations: TFs, transcriptional factors.

Supplementary Files

This is a list of supplementary files associated with this preprint. Click to download.

- FigureS1.tif

- Figures2.tif

- Figures3.tif

- Tables1.xls 